\title{
Clinical trials and future directions in pediatric acute respiratory distress syndrome
}

\author{
Veerajalandhar Allareddy, Ira M. Cheifetz \\ Section Chief Pediatric Cardiac ICU, Duke Children's Hospital, Duke University Medical Center, Durham, NC, USA \\ Contributions: (I) Conception and design: All authors; (II) Administrative support: All authors; (III) Provision of study materials or patients: All \\ authors; (IV) Collection and assembly of data: All authors; (V) Data analysis and interpretation: All authors; (VI) Manuscript writing: All authors; (VII) \\ Final approval of manuscript: All authors. \\ Correspondence to: Veerajalandhar Allareddy, MD, MBA. Section Chief Pediatric Cardiac ICU, Duke Children's Hospital, Duke University Medical \\ Center, Box 3046, 5th Floor, DN, Rm 5260Y PCCM Office Suite, Durham, NC 27710, USA. Email: veera.allareddy@duke.edu.
}

\begin{abstract}
The pediatric acute respiratory distress syndrome (PARDS), a description specific for children with acute respiratory distress syndrome (ARDS), was proposed in the recent Pediatric Acute Lung Injury Consensus Conference (PALICC, 2015). This recent standardization of PARDS diagnosis is expected to aid in uniform earlier recognition of the entity, enable use of consistent management strategies and potentially increase the ease of enrollment in future PARDS clinical trials—all of which are expected to optimize outcomes in PARDS. Clinical trials in PARDS are few but ongoing studies are expected to lay the foundation for future clinical studies. The Randomized Evaluation of Sedation Titration for Respiratory Failure trial (RESTORE) trial has revealed that a goal directed sedation protocol does not reduce the duration of invasive ventilation in critically ill children. PROSpect trial is a large multi-institute clinical trial that is expected to reveal optimal ventilation strategies and patient positioning (supine $v s$. prone) in patients with severe PARDS. The PARDS neuromuscular blockade (NMB) study is expected to yield important information about the impact of active NMB on PARDS outcomes. Information from these studies could be used to design future clinical trials in PARDS and to lessen the anecdotal or extrapolated experiences from adult clinical studies that often guide clinical practices in PARDS management. Finally, it is expected that these definitions and management strategies will be revised periodically as our understanding of PARDS evolves. Emerging data on PARDS subtypes suggest that patient heterogeneity is an important factor in designing these clinical trials.
\end{abstract}

Keywords: Pediatric acute respiratory distress syndrome (PARDS); clinical trials; biomarkers; ventilation strategies; supportive therapies

Submitted Aug 09, 2019. Accepted for publication Aug 30, 2019.

doi: 10.21037/atm.2019.09.14

View this article at: http://dx.doi.org/10.21037/atm.2019.09.14

Available clinical trials in children with acute respiratory distress syndrome (ARDS) are few in number and insufficient to guide consistent clinical practice. Although, there are several reasons for the relative paucity of clinical trials in pediatric acute respiratory distress syndrome (PARDS), the lack of standardized recognition of the entity up until the recent past as well as its lower reported incidence as compared to ARDS in adults are thought to be main reasons. Lack of consistent diagnostic criteria, the multi-modality treatment interventions often used in ARDS management, and the multiple centers needed for adequate enrollment have all limited the conduct of robust clinical trials in this population. Sparse and conflicting pediatricbased evidence exists, and, thus, extrapolation from adult studies and anecdotal experience generally guide clinical practice in managing those with ARDS. 


\section{Definition of PARDS}

Substantial progress has been made in the past few decades to improve the ARDS definition for children. The Pediatric Acute Lung Injury Consensus Conference (PALICC) in year 2015 developed a definition specific for children, and the term PARDS was proposed (1). Furthermore, to promote optimization and consistency of care, several comprehensive recommendations regarding treatment were proposed.

In the original PALICC definition of PARDS, a lower age limit was excluded; however, certain causes of acute hypoxia/hypoxemia such as neonatal pneumonia, sepsis, meconium aspiration syndrome and congenital diaphragmatic hernia, which are all unique to this population were not included. Subsequently, new findings revealed that the consequences of perinatal lung injury due to these causes are not dissimilar to what is seen in PARDS. This new knowledge coupled with the desire to augment multi-disciplinary approach to care has led to the description of ARDS in the neonatal populationthe Montreux definition of neonatal ARDS (2). Universal terminology may help with prompt recognition, accurate and consistent diagnosis, and improved prognostication in PARDS (3). In addition, relative simplification in criteria, such as the use of oxygen saturation index (OSI) when an oxygenation index (OI) is not available or $\mathrm{SpO}_{2} / \mathrm{FIO}_{2}$ ratio when $\mathrm{P} / \mathrm{F}$ ratio is not feasible, for risk stratification of patients with PARDS may aid in earlier recognition and management of this entity. Further, this could potentially increase the ease of enrollment in future clinical trials. It is expected that as our understanding of the etiology, pathophysiology and management of neonates, infants and children with PARDS improves these descriptions will be updated as required.

One of the key aspects of PALICC was to identify areas of uncertainty requiring further investigation. There is an urgent need to foster multi-institute clinical research initiatives tailored to the PARDS population.

\section{Epidemiology and outcomes of PARDS}

When compared to adults, the reported incidence of ARDS in children is relatively low at about 2 to 12.8 per 100,000-person-years (4-6). A recent study has shown that the application of the PALICC definition has led to an increase in the number of children with ARDS being identified. A recent retrospective study of 4,764 hospitalizations in a tertiary care pediatric intensive care unit (PICU) has shown an increase in PARDS diagnosis during the study period [278 children based on PALICC definition group vs. 134 based on the American European Consensus Conference (AECC) definition group vs. 143 based on the Berlin definition group] (7). In this study, the overall mortality had also declined $(5.8 \%$ vs. $30.6 \%$ vs. $32.2 \%$ in PALICC vs. AECC vs. Berlin definition, respectively) (7). In the current PARDS definition, the severity of lung disease is stratified into mild, moderate, and severe groups (1). A multicenter, retrospective, descriptive cohort study of ten multidisciplinary PICUs in Asia showed that PALICC risk stratification accurately predicted outcomes in PARDS. In this study involving 373 children, the in-hospital mortality (IHM) rate had increased gradually according to the severity of the PARDS per PALICC definition. The overall PICU mortality rate was $30.3 \%$ and the 100 -day mortality rate was $39.7 \%$, which is similar to the Berlin and AECC definitions (8).

Large recently published pediatric outcome studies and meta-analysis have revealed an overall mortality rate of $24.0 \%$ to $33.7 \%$ in children with ARDS $(5,9)$. However, it appears that mortality is likely decreasing in trend (9) probably related to several factors, including but not limited to, early recognition, improved understanding of the etiology and pathophysiology of PARDS, and quicker initiation of appropriate available evidenced based management strategies $(1,10)$.

\section{Ventilator strategies in ARDS}

In the past two decades, adults with ARDS were routinely ventilated with lower tidal volumes (VTs) and higher positive-end-expiratory pressures (PEEPs) as this strategy was thought to be "lung protective" (11-15).

\section{VTs and outcomes}

Historically, before the lung protective strategy, adults with ARDS were managed with high VT of $10-15 \mathrm{~mL} / \mathrm{kg}$ of ideal body-weight (IBW) to achieve optimal ventilation ( $\mathrm{pH}$ and $\mathrm{PaCO}_{2}$ ) and improve oxygenation. Subsequently, several studies have shown the use of such high VT and pressures are associated with worse outcomes. Specifically, these high VTs and pressures caused destruction of the respiratory endothelium which released a variety of inflammatory mediators. Further proliferation of the proinflammatory cascade led to damage of other organs as well 
$(16,17)$. As prior animal studies had shown that invasive mechanical ventilation (MV) with large VTs and pressures can be harmful to the respiratory system (and other organs), limiting the airway pressures and volumes was thought to improve outcomes. To this effect, a multicenter, randomized clinical trial (RCT) was conducted to compare a ventilation strategy that aimed at limiting the end-inspiratory plateau pressure (PP) to $25 \mathrm{cmH}_{2} \mathrm{O}$ and using VT below $10 \mathrm{~mL} / \mathrm{kg}$ of IBW, versus a more traditional ventilatory approach of using VTs at $\geq 10 \mathrm{~mL} / \mathrm{kg}$ and aiming for near normocapnia. The same level of PEEP was used on both arms of the study. In this study, 116 adults with ARDS and no other organ injury were enrolled. This approach did not decrease the duration of $M V$ in days $(23.1 \pm 20.2$ vs. $21.4 \pm 16.3)$, or reduce the IHM rate at day $60(46.6 \%$ vs. $37.9 \%$ in controls). Further, no reduction in the rate of secondary occurrence of multiple organ failure was noted $(41 \%$ vs. $41 \%)$. It was concluded that a strategy targeting reduced VT titrated to reach PPs of $25 \mathrm{cmH}_{2} \mathrm{O}$ was no better than the conventional strategy (11).

Similarly, around that time the pressure and volume limited ventilation strategy trial was conducted (12). Patients at high risk for the ARDS were randomly assigned to either volume and pressure limited ventilation (limitedventilation group), with the peak inspiratory pressure (PIP) maintained at $\leq 30 \mathrm{cmH}_{2} \mathrm{O}$ and $\mathrm{VT}$ at $\leq 8 \mathrm{~mL} / \mathrm{kg}$, or to the conventional ventilation [control group (CG)], with the PIP allowed to rise as high as $50 \mathrm{cmH}_{2} \mathrm{O}$ and the VT at $10-15 \mathrm{~mL} / \mathrm{kg}$. In a total of 120 such patients, the strategy of MV that limits PIP and VT did not appear to reduce IHM and suggested an increase in overall morbidity (12). However, these trials were limited by lesser number of patients enrolled and a greater difference in VT between the two groups.

In a landmark ARDS Network trial (13), participants with acute lung injury (ALI) and ARDS, MV with a lower VT than is conventionally used resulted in a decreased IHM and increased the number of ventilation free days (VFDs). In this multicenter, RCT, an initial VT of $12 \mathrm{~mL} / \mathrm{kg}$ and an airway PP of $\leq 50 \mathrm{cmH}_{2} \mathrm{O}$ was compared with VT of $6 \mathrm{~mL} / \mathrm{kg}$ and a PP of $\leq 30 \mathrm{cmH}_{2} \mathrm{O}$. After enrollment of 861 patients, the trial was stopped because the IHM rate was lower VT group compared to their counterparts $(12 \mathrm{~mL} / \mathrm{kg})$ (31.0\% vs. 39.8\%, $\mathrm{P}=0.007)$. Furthermore, the number of VFDs during the initial 28 days was higher in the lower VT/PP group. The lower VT group also had fewer nonpulmonary organ failure days.

\section{VTs in PARDS}

The effects of VT in PARDS remains unclear. Current pediatric clinical practices, in general, are based on data extrapolated from adult experiences or studies. Routine ventilation strategies in PARDS do not usually involve high VTs as was used in the ARDS Network trial groups $(12 \mathrm{~mL} / \mathrm{kg})$. As such, a similar trial between high and low VTs in children with PARDS may be difficult to design and conduct. A prior multicenter study, across 10 PICUs from Asia that included 373 children with PARDS did not reveal significant IHM rates in participants who were ventilated with VTs in $5-10 \mathrm{~mL} / \mathrm{kg}$ range (7). In any mechanically ventilated pediatric patient, the use of VTs below or within the range of normal physiologic VTs adjusted for the age and body weight (i.e., $5-8 \mathrm{~mL} / \mathrm{kg}$ ) in controlled ventilation strategy according to respiratory system compliance (CRS) and the underlying lung pathology is recommended (1). Further, patient-specific VTs according to severity of disease should also be considered. For example, VTs should be 3 to $6 \mathrm{~mL} / \mathrm{kg}$ IBW for those with reduced respiratory compliance and probably closer to the expected or normal physiologic range (5 to $8 \mathrm{~mL} / \mathrm{kg}$ IBW) for those with better preserved respiratory compliance. In the absence of advanced transpulmonary pressure (TPP) measurements, it is recommended that an inspiratory $\mathrm{PP}$ limit of $28 \mathrm{cmH}_{2} \mathrm{O}$ be considered; however, on occasions slightly higher PPs (29 to $32 \mathrm{cmH}_{2} \mathrm{O}$ ) could also be considered for those with increased chest wall elastance (decreased chest wall compliance) on an individual basis. It is important to understand that all of these recommendations are based on weak agreement amongst the consensus experts (1). To systematically address these issues carefully designed RCTs that evaluate the impact of VTs/PPs in PARDS are urgently required.

\section{Driving pressures and outcomes}

As discussed earlier, lung-protective ventilation strategy recommends the use of low VTs, lower end-inspiratory (plateau) pressures and higher levels of PEEP. However, it is known that reducing VT does not always prevent overstrain or overstress. Conceptually, titrating MV on airway driving pressure, computed as airway pressure changes from PEEP to end-inspiratory PP, equal to the ratio between the VT and compliance of pulmonary system, may better reflect lung injury. In a small study of 150 adult ARDS patients, those with the higher airway driving pressures $\left(\geq 15 \mathrm{cmH}_{2} \mathrm{O}\right)$ 
had a considerably higher lung stress, respiratory system and lung-elastance compared to their counterparts. Airway driving pressure was significantly related to lung stress [at $5 \mathrm{cmH}_{2} \mathrm{O}$ PEEP: $\mathrm{r}(2)=0.581, \mathrm{P}<0.0001$; at PEEP of $\left.15 \mathrm{cmH}_{2} \mathrm{O}: \mathrm{r}(2)=0.353, \mathrm{P}<0.0001\right]$. For a lung stress of 24 and $26 \mathrm{cmH}_{2} \mathrm{O}$, the optimal cutoff value for the airway driving pressure were 15.0 [receiver operating characteristic (ROC)-area under the curve (AUC), 0.85; 95\% confidence interval (CI), 0.782-0.922]; and $16.7 \mathrm{cmH}_{2} \mathrm{O}$ (ROCAUC, 0.84; 95\% CI, 0.742-0.936) (18). To further address the relative importance of VT, PEEP and plateau airway pressures, the driving pressures $(\triangle \mathrm{P}=\mathrm{VT} / \mathrm{CRS})$ were examined as an independent variable related with survival. In an observational, multilevel mediation analysis of 3,562 adults with ARDS enrolled in 9 previously reported RCTs, the $\Delta \mathrm{P}$ was most strongly associated with survival. A 1 standard deviation increment in $\Delta \mathrm{P}$ (approximately $7 \mathrm{cmH}_{2} \mathrm{O}$ ) was associated with increased IHM [relative-risk (RR) of $1.41 ; \mathrm{P}<0.001]$, even in participants who received "protective" PP and VTs (RR, 1.36; $\mathrm{P}<0.001)$. Decreases in driving pressures were strongly associated with increased survival (19). Since this is an observational study, no causation can be inferred. Future RCTs are needed to assess the impact of driving pressure in those with ALI/ARDS. Clinical researches pertaining to effect of driving pressures in PARDS are lacking and should be the focus in future studies.

\section{PEEP and outcomes}

The optimal level of PEEP to be used in the management of adults with ARDS remains elusive $(14,15,20,21)$. Providers should be aware that the deleterious effects of higher or excessive PEEP may actually outweigh the potential benefits. It is well known that the end-inspiratory alveolar over-distention resulting from using high PEEP increases the pulmonary vascular resistance (PVR) and alveolar dead space and thus worsens the intrapulmonary shunt $(22,23)$. The optimal PEEP level in PARDS is unknown and current practices are mainly inferred from the adult data (14).

The effects of high PEEP depends to a significant extent on the "lung recruitability", which unfortunately fluctuates extensively in ALI/ARDS. This variation is thought to exist within a patient and between patients, partly again related to the severity of the disease process. Data on 68 participants who had ALIR or ARDS and had a lung computed tomography (CT) scan performed at three different levels of airway pressure including 5, 15 and $45 \mathrm{cmH}_{2} \mathrm{O}$ were investigated. Only in those participants with a higher percentage of recruitable lung tissue, the increments in PEEP showed potential beneficial effects. In addition, as expectedly, a corresponding increase in alveolar strain was noted in the two groups. Opening and closing lung tissue was an independent risk factor for IHM [odds ratio (OR) was 1.1 for every 10 -gram increase]. The hilar and the dependent regions of the lungs were more subject to the effects of opening and closing lung tissue (24). It was concluded that the favorable effects of decreasing intra-tidal alveolar opening and closing by increasing PEEP prevailed over the deleterious effects of increasing the alveolar strain.

A large systematic Cochrane register based review of 2,565 adult participants with ALI and ARDS across seven RCTs compared the effects of two levels of PEEP. High levels of PEEP as compared to low levels of PEEP improved the participant's oxygenation at days 1, 3 and 7; however, it did not decrease the overall IHM or significantly increase the risk of barotrauma (14). Clinical heterogeneity was a major limitation to this study.

Higher PEEP use compared to lower PEEP does not lead to favorable survival (15). In this study, individual data from 2,299 adults across 3 clinical trials were analyzed. In adults with ARDS $(n=1,892)$, the higher PEEP group had an IHM rate of 34\% (324 deaths) as compared to 39\% (368 deaths) in the lower PEEP group [absolute risk reduction (ARR), 0.90]. In the same study, in those participants without ARDS $(\mathrm{n}=404)$, the higher PEEP group had an IHM rate of $27.2 \%$ (50 deaths) vs. 19.4\% (44 deaths) in the lower PEEP group (ARR, 1.37). Further, in a subgroup of these adult patients with moderate-severe ARDS, the use of higher PEEP was associated with improved survival.

A large systematic review and meta-analysis of 8 RCTs involving 2,728 participants, investigating MV strategies using higher (mean $15 \mathrm{cmH}_{2} \mathrm{O}$ ) versus lower (mean $9.1 \mathrm{cmH}_{2} \mathrm{O}$ ) PEEP levels among unselected participants with ARDS revealed that the use of higher PEEP was not associated with improved outcomes (20). In this study, a random effects model was used to evaluate the impact of higher PEEP on 28-day mortality, VFDs, oxygenation and ventilation, barotrauma and organ failure. Primary analysis did not reveal significantly reduced IHM for patients receiving higher PEEP compared to their counterparts (i.e., lower PEEP group) (six trials; 2,580 patients; RR, 0.91; 95\% CI, 0.80-1.03). Two trials that did not use lower VT in the lower PEEP CGs were excluded. Further, a higher PEEP strategy also did not significantly decrease VFD, barotrauma or new organ failure compared to the lower PEEP strategy. 
A recent large multicenter [120 intensive care units (ICUs) from nine countries], RCT of 1,010 adults with moderate-severe ARDS was conducted to evaluate if lung recruitment with PEEP titration (use of higher PEEP) based on the best pulmonary compliance decreased the 28-day IHM rate compared to a conventional strategy of using lower PEEP. Pertinent other outcomes were ICU and hospital length of stay; VFDs through initial 28 days, complications such as barotrauma within 7 days or pneumothorax requiring chest tube; and mortality in ICU, in-hospital, or at 6 months (21). Fifty-five point three percent in the experimental group (EG) and $49.3 \%$ in the CG had died at 28 days [hazard ratio (HR), 1.20; $\mathrm{P}=0.041$ ]. Patients in the EG had an increased 6 month mortality (65.3\% vs. 59.9\%; HR, 1.18; $\mathrm{P}=0.04$ ), a decreased number of VFD (5.3 vs. 6.4; difference, $-1.1 \% ; \mathrm{P}=0.03$ ), an increased risk of pneumothorax requiring chest tube $(3.2 \%$ vs. $1.2 \%$; difference, $2.0 \% ; \mathrm{P}=0.03$ ), and higher risk of barotrauma (5.6\% vs. $1.6 \%$; difference, $4.0 \%$; $\mathrm{P}=0.001$ ). However, there was no statistically significant difference in the length of stay (hospital or ICU), or mortality (hospital or ICU). Routine PEEP titration maneuvers are not recommended (21).

Based on these various systematic reviews and metaanalyses, higher PEEP may improve oxygenation in certain subsets of ARDS population, however, high PEEP is not associated with significant survival benefit. Further, it appears that the advantages of higher PEEP levels are more obvious in those participants with moderate-severe ARDS. A more recent ARDS trial (ART) investigators study, a RCT (21), provides further clinical equipoise on the benefit of higher PEEP in those with ARDS. Higher IHM rate was seen in those who were managed with higher PEEP, and thus routine PEEP titration/lung recruitment maneuvers that use higher PEEPs is not recommended in adults.

\section{PEEP in PARDS}

At this time, the optimal PEEP in children with PARDS is unknown. As none of the available RCTs and metaanalyses included children, it is unclear if higher PEEP offers any clinical advantage in PARDS. In a single center, retrospective study the management of $M V$ between two eras was compared [past group (PG): year 1998-1992, N=79 vs. recent group (RG): year 2000-2004, $\mathrm{N}=85]$ (25). In this study, children in the PG were ventilated with a higher mean VT, higher mean PIPs, and lower levels of PEEPs as compared to the RG patients. The patients in the RG had a significantly lower IHM rate (21\% vs. $35 \%)$ and a greater number of VFDs. A higher VT was also significantly associated with both increased IHM rate (OR, 1.59) and reduction in VFDs. A $40 \%$ reduction in mortality was observed. In this study, thus lower VT was associated with decreased IHM and an increase in the number of VFDs. However, single center and retrospective design limit the generalizability of these results.

For those with severe PARDS, higher PEEP levels $>15 \mathrm{cmH}_{2} \mathrm{O}$ may be needed; however, PPs should be limited to the minimal extent as tolerated (strong agreement amongst the PALICC experts). Further, in those with severe PARDS, up titrating the levels of PEEP from 10 to up to $15 \mathrm{cmH}_{2} \mathrm{O}$ (moderately elevated) to optimize oxygenation should include associated close observation of the hemodynamic response (weak agreement amongst the experts). Cautious slow incremental or decremental PEEP recruitment maneuvers may also be considered in those with severe oxygenation failure (weak agreement) (1).

The optimal PEEP in PARDS remains to be fully studied. On occasions, PEEP $>15 \mathrm{mmHg}$ might be required in severe PARDS, if so, monitoring of pulmonary compliance and bedside hemodynamics is highly recommended. In addition, these patients are at higher risk for barotrauma/pneumothorax. Future clinical trials should focus on the impact of varying levels of PEEP in varying severity of PARDS.

Apart from the above MV strategies, currently there is no data on the impact of mode of ventilation (control $v s$. assisted) on outcomes in PARDS. This should be the focus in future studies.

\section{High-frequency ventilation}

The OSCAR study group showed that in adults with ARDS the use of high-frequency oscillatory ventilation (HFOV) did not significantly effect the 30-day IHM rates (26). In this multicenter study, adults with $\mathrm{ARDS}\left(\mathrm{PaO}_{2} / \mathrm{FiO}_{2}\right.$ $\leq 200 \mathrm{mmHg}$ ) were randomly assigned to undergo either HFOV or routine conventional ventilatory care. After adjustment for several confounders, there was no significant survival benefit between the two groups (conventional ventilation group with OR of $1.03 ; 95 \%$ CI, $0.75-1.40 ; \mathrm{P}=0.87$ ).

The early use of HFOV when compared to the conventional strategy of low VT and high PEEP may lead to worse outcomes in adults with moderate to severe ARDS. This was shown in a large, multicenter, randomized, controlled trial that was conducted in 39 ICUs across five countries. In this study, adults with moderate to severe 
ARDS were assigned to either HFOV or to a ventilation strategy that used low VT and high PEEP (the conventional group) (27). All cause IHM rate was the primary outcome of this study. The trial was stopped after 548 of the 1,200 patients were enrolled since the interim analysis showed that the IHM rate was higher in the HFOV group ( $47 \%$ vs. $35 \%$ in the conventional group). The RR of IHM with use of HFOV was 1.33 (95\% CI, 1.09-1.64; $\mathrm{P}=0.005$ ).

In moderate to severe PARDS, it is recommended that HFOV be considered in those with PPs $>28 \mathrm{cmH}_{2} \mathrm{O}$ and there is no evidence of decreased chest wall compliance (1) (weak agreement). In doing so, the optimal lung volume should be achieved by recruitment maneuvers that use gradual increase or decrease of the Paw (continuous distending pressures). During this process, serial bedside hemodynamic assessments and monitoring of the $\mathrm{CO}_{2}$ and oxygenation are needed (1). Further studies are needed to strengthen these recommendations (see below for PROSpect trial).

\section{Pulmonary specific ancillary treatment}

\section{Prone positioning}

Prone positioning is thought to improve alveolar-capillary gas exchange by decreasing the dorsal lung compression, improving the ventral-dorsal TPP difference, and optimizing the lung perfusion. Prior RCTs and metaanalyses initially reported no mortality benefit with prone positioning for adults with ARDS (28-35). However, the benefit of prone ventilation in a sub-population of adults with severe ARDS who were MV with low VTs was revealed by the PROSEVA trial. This was a multicenter, randomized trial, of early (within 33 hours of intubation), high-dose (17 consecutive hours) prone ventilation in those with severe ARDS (36). This trial of 466 patients receiving low VT for severe ARDS $\left(\mathrm{PaO}_{2}: \mathrm{FiO}_{2}<150 \mathrm{mmHg}, \mathrm{FiO}_{2} \geq 0.6\right.$, PEEP $\geq 5 \mathrm{cmH}_{2} \mathrm{O}$, a VT close to $6 \mathrm{~mL} / \mathrm{kg}$ of IBW), revealed that those receiving prone ventilation (average time spent prone: $73 \%)$ had a reduction in 28 -day IHM (16\% vs. $33 \%$; HR, 0.39; 95\% CI, 0.25-0.63) and 90-day IHM (24\% vs. 41\%; HR, 0.44; 95\% CI, 0.29-0.67) compared to their counterparts. Further, patients in the prone group needed less rescue therapy including inhaled nitric oxide (iNO) $(10 \%$ vs. $16 \%)$ or extracorporeal membrane oxygenation (ECMO) (1\% vs. 26\%) (36). Important limitations to this study included the highly selective group (minority of patients with ARDS), several exclusionary criteria [elevated intracranial pressure (ICP), mean arterial blood pressure (BP) $<65 \mathrm{mmHg}$, pneumothoraces, etc.] and non-matching of patients despite randomization. These factors preclude uniform generalizability.

At this time, routine prone positioning is not recommended in PARDS (1). However, pending results of the PROSpect trail, it could be considered as an option in select cases of PARDS. The PROSpect trial is a two by two factorial, response adaptive, RCT of supine/prone patient positioning and use of conventional MV (CMV) or HFOV (37). It is an international multicenter trial involving about 45 PICUs, across 30 US and 15 international centers. Up to 1,000 subjects with severe PARDS are to be enrolled and stratified by age group and by type of lung injury (direct/indirect). The PROSpect trial began enrollment in May 2019 and is expected to be completed by May of 2024. Inclusion criteria would include those children $\leq 18$ years who are invasively ventilated for severe PARDS for $<48$ hours per PALICC recommendations (chest $\mathrm{X}$-ray suggesting acute pulmonary parenchymal disease and OI $\geq 16$ or OSI $\geq 12.3$ ). Further, 2 arterial blood gases that suggest severe PARDS are required. These blood gases are to be separated by $\geq 4 \pm 2$ hours during which time the provider team is actively attempting to recruit/optimize lung volume while simultaneously optimizing the participant's hemodynamic status. this specifically, entails the use of incremental and decremental PEEP changes to optimize lung volume). Participants will be in prone position for $\geq 16$ hrs per day (maximum of 28 days). The CMV strategy includes low VT to obtain VT of $5-7 \mathrm{~mL} / \mathrm{kg}$ (IBW), PIP target to $\leq 28 \mathrm{cmH}_{2} \mathrm{O}$, and a pulmonary recruitment strategy to find the optimal PEEP and subsequently maintained per a $\mathrm{PEEP}-\mathrm{FiO}_{2}$ grid. The HFOV strategy would include $8-12 \mathrm{~Hz}$ of frequency, $60-90$ of delta-P (amplitude), and mPaw lung recruitment maneuvers. The PROSpect trial's overall objective is to identify the optimal ventilation strategy or patient position that improves overall outcomes in severe PARDS. In this trial, eligible consecutive subjects with severe PARDS will be randomized to 1 of 4 predefined groups: CMV/supine, CMV/prone, HFOV/supine, and $\mathrm{HFOV/prone.} \mathrm{Those} \mathrm{who} \mathrm{fail} \mathrm{their} \mathrm{ventilation} \mathrm{strategy}$ or positioning for either persistent or worsening hypoxia or hypercarbia could receive the reciprocal therapy while being considered for extracorporeal life support. The main outcome of this trail is VFDs through day 28. The nonsurvivors receive zero VFDs. Non-pulmonary organ failure free (OFF) days is a secondary outcome of interest. The study will further explore the impact of prone positioning 
with HFOV on VFDs and also investigate the effects of these interventions on outcomes such as 90-day IHM, and the duration of MV, length of stay (PICU and hospital), and the path of post ICU functional status including the healthrelated quality of life (HRQL) (37).

\section{iNO}

iNO is not considered routine therapy for adults with ARDS. Although, iNO use improves oxygenation, it has not been shown to reduce IHM, duration of MV or VFDs; and may be associated with higher risk of renal impairment (38-45).

Due to the lack of well-designed clinical trials in PARDS, routine use of iNO is not recommended in clinical practice (1). However, iNO could be considered in select cases of severe PARDS, mainly as a bridge to recovery, decision making or as rescue therapy from ECMO. The initiation or maintenance dose, the duration and subsequent weaning pathways are not well established in PARDS. Wide variation in the use of $\mathrm{iNO}$ exists in pediatric clinical practice. Future studies are needed to address these uncertainties.

\section{Steroids and exogenous surfactant}

Routine use of corticosteroids and/or exogenous surfactant therapy is not recommended in PARDS management (1). An important limitation of the data on glucocorticoid therapy in adults with ARDS is that low VT (lung protective strategies) was not consistently performed in the majority of prior clinical trials. Two recent trials $(46,47)$ that documented use of low VT strategies reported similar mortality rates among adult patients receiving glucocorticoids compared with placebo. Further studies in PARDS should focus on specific patient populations (severity, subsets of PARDs, type of injury, etc.) who might benefit from steroid and/or surfactant therapy (1).

\section{Non-pulmonary treatments}

\section{Neuromuscular blockade (NMB)}

In adults with ARDS the role of paralysis (NMB) is unclear. This is largely driven by competing effects of NMB on patient outcomes based on two large trials.

The first was the European ACURASYS trial (48). In this multicenter, placebo controlled trial, 340 adults with an acute onset of severe ARDS (within 48 hours) were assigned to receive, for 48 hours, either NMB (cisatracurium) or placebo. In this study, severe ARDS was defined as a $\mathrm{PaO}_{2}: \mathrm{FIO}_{2}$ of $<150$ with PEEP $\geq 5 \mathrm{cmH}_{2} \mathrm{O}$ and VT of 6-8 mL/kg (adjusted to IBW). Both groups were deeply sedated. After adjustment for several confounders, the HR for death at 90 days in the cisatracurium group was $0.68(\mathrm{P}=0.04)$. Patients treated with NMB had nonstatistically significant lower crude 90-day, 28-day, hospital, and ICU mortality rates compared to their counterparts. The beneficial effects on 90-day mortality were limited to patients who presented with severe ARDS $\left(\mathrm{a}^{\mathrm{PaO}_{2}}: \mathrm{FiO}_{2}\right.$ $<120$ ). In addition, those treated with cisatracurium also had significantly more VFDs during the first 28 and 90 days and were significantly less likely to experience complications (barotrauma).

The second major RCT was the recently concluded ROSE trial (49). The benefits of early continuous NMB in adults with ARDS who received invasive MV was assessed. Adult participants with moderate-to-severe ARDS (defined by $\mathrm{PaO}_{2}: \mathrm{FiO}_{2}<150$ with a PEEP $\geq 8 \mathrm{cmH}_{2} \mathrm{O}$ ) were randomly assigned to an interventional group (IG) (48-hour continuous infusion of cisatracurium) or to a CG (usual care without routine NMB and lighter sedation). The same MV strategies were used in both groups. At the second interim analysis this study was discontinued for futility. The IHM rate at 90 days, was $42.5 \%$ (213 patients) in the IG and $42.8 \%$ (216 patients) in the CG (between-group difference, $-0.3 \%$ points; $95 \% \mathrm{CI},-6.4$ to $5.9 ; \mathrm{P}=0.93)$. Further, there were no between group differences in several end points assessed at 3, 6, and 12 months.

The PARDS NMB study (PAN, ClinicalTrials.gov Identifier: NCT02902055, "Life-threatening acute respiratory failure in children: to breathe or not to breathe spontaneously, that's the question") is a phase 4, randomized, placebo controlled, prospective, interventional clinical trial that aims to assess the impact of active NMB with rocuronium (vs. isotonic saline) on PARDS outcomes (50). Children up to 5 years of age with early moderate to severe PARDS of any cause and needing $M V$ with TV $(5-8 \mathrm{~mL} / \mathrm{kg}$ IBW) and PEEP equal to or greater than $5 \mathrm{cmH}_{2} \mathrm{O}$ will be the participants. The main outcome of this study is the cumulative respiratory morbidity score 12 months after PICU discharge (50).

In PARDS, if sedation by itself is not adequate to attain effective MV, then paralysis with NMB should be considered. If so, most minimal yet effective paralysis with adequate sedation to facilitate their tolerance to MV (including patient ventilator synchrony) and/or to 
optimize demand (i.e., decrease the work of breathing and consumption) should be considered. In children with PARDS, the short- and long-term sequelae/outcomes of NMB use should be studied. For future clinical trials, the NMB drug type, dosage, strategies (bolus vs. drips), goals and weaning pathways should be adequately defined. Likewise, monitoring strategies (example train of fours) should be well detailed and explicit so as to allow comparison across clinical studies (1).

\section{Sedation}

Protocolized sedation is thought to improve clinical outcomes in critically ill adults. The Randomized Evaluation of Sedation Titration for Respiratory Failure trial (RESTORE), a multicenter cluster randomized trial of 2,449 children in 31 US PICUs, assessed whether critically ill children managed with a nurse-implemented, goal-directed sedation protocol experienced fewer days of $M V$ than patients receiving usual care (51). In this study, 1,225 children were subject to a targeted sedation, arousal assessments, extubation readiness testing, sedation adjustment every 8 hours, and sedation weaning. In this study, amongst children who underwent MV for acute respiratory failure, the use of a sedation protocol compared to routine care did not reduce the duration of MV (51).

In PARDS, children should receive adequate sedation to assist their tolerance to MV. Simple, reliable and valid pain/sedation scales appropriate for different ages and adjusted for weight should be used for monitoring and titrating to achieve sedation goals. These should assist interprofessional communication (decrease provider variability in interpretation). Future clinical studies in PARDS should report specific pain/sedation goals and strategies to achieve optimal outcomes. This should also allow for adequate comparison across studies.

\section{Fluid management}

Optimal fluid balance management strategy in patients with ARDS is unclear in both adults and pediatric population. In a randomized study, a conservative [pulmonary artery occlusion pressure $(\mathrm{PAOP})<8 \mathrm{mmHg}$ or central venous pressure $(\mathrm{CVP})<4 \mathrm{mmHg}$ ] vs. liberal strategy PAOP 14-18 mmHg or CVP $10-14 \mathrm{mmHg}$ ) of fluid management was applied for 7 days in 1,000 adults with ALI. Overall, there was no difference in the 60-day IHM rate between the two groups; however, the conservative strategy group
$(-136 \mathrm{~mL})$ had an improved OI and lung injury score, with increasing VFDs (15 vs. 12 days) and ICU-free days (13 vs. 11 days) compared to the liberal strategy group $(+6,992 \mathrm{~mL})(52)$.

In PARDS, after initial fluid resuscitation and stabilization, an objective targeted (net balance) fluid management strategy that includes total fluid intake and output should be considered. Total volume balance should be frequently assessed and titrated to goal so as to maintain adequate intravascular volume and hemodynamics $(1,10)$. Insensible losses should be factored into the decisionmaking process while having well defined goal balances. Future clinical studies are required to determine the best fluid management strategy in PARDS. Clinical protocols should guide daily fluid management goals. The clinical trials should use easily adaptable protocols that also allow adequate comparison between studies (e.g., indication for fluid boluses, amount and type of fluid, duration of replacement, use of established monitoring such as CVPs). In addition, early markers of renal injury should be incorporated into clinical practices (example, use of NGALs or other objective measures).

\section{Conservative vs. liberal oxygenation targets}

In the acutely ill adults, the Improving Oxygen Therapy in Acute-illness (IOTA) study revealed that liberal oxygen therapy increases IHM rate without improving relevant outcomes. In the IOTA study which is a systematic review/ meta-analysis, 25 RCTs enrolled a total of 16,037 adults with a variety of acute illnesses, including but not limited to sepsis, trauma, stroke, myocardial infarction, cardiac arrest, and status-post surgical procedures. The liberal oxygen strategy [median baseline $\left(\mathrm{SpO}_{2}\right)$ across trials of 96\%; range, 94-99\%, interquartile range (IQR), 96-98] increased IHM rate [RR, 1.21; 95\% CI, $1.03-1.43 ; \mathrm{I}^{2}=0 \%$; high quality (HQ)], at 30 days (RR, 1.14; 95\% CI, 1.01$1.29 ; \mathrm{I}^{2}=0 \% ; \mathrm{HQ}$ ), and at longest follow-up as well (RR, 1.10 ; $95 \% \mathrm{CI}, 1.00-1.20 ; \mathrm{I}^{2}=0 \%$; HQ) compared to the conservative strategy (53). The Oxy-PICU trial showed that a trial of peripheral $\mathrm{O}_{2}$ saturation $\left(\mathrm{SpO}_{2}\right)$ targets is feasible in critically ill children (54). The Oxy- PICU was an open, randomized, parallel group trial of children $>38$ weeks and $<16$ years of age who were admitted urgently to PICUs and received either non-invasive or invasive respiratory support including supplemental $\mathrm{O}_{2}$. Children were assigned to a liberal $\mathrm{O}_{2}$ group $\left(\mathrm{SpO}_{2}\right.$ target $\left.>94 \%\right)$ or a conservative $\mathrm{O}_{2}$ group $\left(\mathrm{SpO}_{2}=88-92 \%\right.$ inclusive). Outcomes were measures of feasibility including recruitment rate, protocol adherence 
and acceptability, between group separation of $\mathrm{SpO}_{2}$ and safety. In this study, there were no statistically significant between-group differences in duration of organ support, IHM or length of stay.

\section{Other novel approaches to targeted MV}

In a small randomized trial of 61 adults with ALI or ARDS who underwent invasive MV with PEEP adjusted per targeted measurements of esophageal pressures (EPs) to estimate the TPPs (EP guided group) or per the ARDS Network standard-of-care (CG), it was noted that the EP guided group had significantly improved compliance and oxygenation compared to their counterparts. The ratio of the $\mathrm{PaO}_{2}: \mathrm{FIO}_{2}$ at 72 hours was $88 \mathrm{mmHg}$ higher in the EP group (95\% CI, 78.1-98.3; P=0.002). Further, this beneficial effect was present over at 24,48 , and 72 hours. Likewise, the CRS was also better at 24, 48, and 72 hours in the EP guided group (55). The Esophageal Pressure-Guided Ventilation 2 Trial (EPVent2) is an adult, multicenter, prospective, randomized, phase II clinical trial that aims to assess the impact of $M V$ directed at sustaining a positive transpulmonary pressure (PTP) in participants with moderate-to-severe ARDS (56). This study is expected to enroll 200 participants from 11 hospitals across North America. The trial will test if the use of a PTP guided ventilation strategy (vs. high PEEP strategy) will lead to improvement in outcomes such as IHM and VFDs at 28 days.

\section{Patient heterogeneity, biomarkers and personalized medicine in PARDS-looking ahead}

It is well known that certain therapies that have been effective in pre-clinical studies or in a specific subset of patients often are proven to be ineffective in a larger cohort trial. Patient heterogeneity exists amongst patients with ARDS and this may lead to varying results. This heterogeneity could be related to the etiology, timing of clinical presentation, intrinsic response of the body systems to the insult, and the timing and duration of the current standard therapy. If we are able to identify or risk stratify the ARDS cohorts based on specific biomarkers or signatures, preferably in a timely fashion, then the likelihood of a clinical study showing no statistically significant differences between the control and treatment groups could be diminished. A variety of biomarkers of ARDS exist (57-60); however, the most appropriate clinically relevant panel of investigations is unclear. In future, personalized medicine approach of identifying ARDS phenotypes based on their unique etiology may enable targeted management of individual or groups with similar ARDS signatures.

Clinical and biological information from two adult ARDS RCTs (the ARMA trial and the ALVEOLI trial) were used to identify such sub-phenotypes of ARDS. The relationship between the sub-phenotypes and clinical outcomes was assessed. In addition, the responses to varying levels of PEEP in the ALVEOLI cohort were studied. Of the 1,022 adults with ARDS (473 in the ARMA and 549 in the ALEVOLI studies), the independent latent class models showed that a 2-class model (i.e., 2 sub-phenotype) was the optimal fit for both populations. In both groups, phenotype 2 (the hyper-inflammatory sub-phenotype) was characterized by a higher prevalence of vasopressor use, higher plasma concentrations of inflammatory biomarkers, lower $\mathrm{HCO}^{-}$, and higher sepsis prevalence than the phenotype 1 . Patients with this phenotype 2 had higher IHM rate, lower VFDs and fewer OFF days in both populations compared to phenotype 1 . In the ALVEOLI group, the impact of ventilation strategy (high $v$ s. low PEEP) on IHM, VFDs and OFF days differed significantly by the type of phenotype ( $\mathrm{P}=0.049$ for IHM, $\mathrm{P}=0.018$ for VFDs, $\mathrm{P}=0.003$ for OFF days) (57).

The underlying etiology of ARDS appears to play an important role in overall outcomes. Lung injury can be divided into direct and indirect molecular phenotypes. In the former, the lung injury due to pneumonia or aspiration leads to severe lung epithelial injury; whereas, in the latter, as for example resulting from non-pulmonary sepsis, a less severe endothelial injury results. Serum biomarkers of pulmonary epithelial and endothelial cell injury exist. In two separate adult studies (100 adults with ARDS/Severe sepsis and 853 adults with ARDS from a multicenter RCT) the plasma biomarkers of pulmonary epithelial and endothelial cell injury were measured. Participants with "direct" ARDS had significantly higher levels of surfactant protein D (biomarker of lung epithelial cell injury) and significantly lower levels of angiopoietin-2 (biomarkers of endothelial cell injury) when compared to patients with "indirect" ARDS. Even after adjustment for severity of illness and severity of ARDS, these correlations were robust. In the multicenter study, participants with direct ARDS had lower levels of both von Willebrand factor antigen (biomarker of endothelial cell injury) and interleukin-6 (IL-6)/IL-8 (inflammatory markers) (58). This information is important if appropriate targeted therapy is being considered based on etiology. 
Recently, it was shown that subtypes of PARDS exist and that they have different predictors of mortality (61). In a prospective cohort study of 544 children with ARDS (Berlin definition), participants were stratified into direct $v s$. indirect ARDS, and also separated into infectious $v s$. non-infectious ARDS. Children with direct ARDS had worse oxygenation but lower severity of illness compared to their counterparts. The predictors of mortality were similar for both direct and indirect ARDS in this cohort. Infectious ARDS had worse oxygenation, lower severity of illness, and lower mortality. In the multivariable analysis, immunocompromised status showed effect modification between infectious and non-infectious ARDS, with no association with IHM in non-infectious ARDS.

In the RESTORE trial, plasma IL- 8 levels were measured within 24 hours of consent and again at 48 hours later. The RESTORE is a multi-center prospective cohort study of hospitalized children aged 2 weeks to 17 years with acute airway or parenchymal disease. The study enrolled children with PARDS across 22 PICUs. In this study, plasma IL-8 levels were strongly associated with severity of illness and oxygenation defect. In addition, the IL-8 levels were consistently higher in patients with PARDS compared to their counterparts; the IL-8 levels were also 4-12 fold higher in non-survivors. IL-8 level was independently associated with IHM, duration of MV, and PICU length of stay on all days measured. However, elevated IL-8 was not associated with PARDS development (60).

Identification of PARDs subtypes using gene expression profiling of body tissue(s) could enable risk stratification, improve targeted therapy and optimize outcomes. The Identifying PARDS Endotypes clinical trial (NCT03539783) is a prospective, observational, casecontrol study that seeks to identify important PARDS subtypes using gene expression profiling of nasal and bronchial epithelial cells from endotracheally intubated children with PARDS (62). In this study, the brushing RNA will be processed by microarray for gene expression analysis and compared to previously published serum biomarkers such as IL-8, angiopoietin-2, and advanced glycosylation end-product specific receptor to assess if there is any correlation and to identify any PARDS endotypes. Changes in gene expression over time will also be assessed to define a PARDS recovery gene expression signature (62). This study is expected to enroll 30 children who are $>5 \mathrm{~kg}$ and $<18$ years of age with PARDS and expected duration of endotracheal intubation $\geq 4$ days and compare the outcomes with controls (Children without PARDS). Outcomes of interest in this study include the determination of pathways and processes that differentiate PARDS recovery from nonrecovery as assessed by improvement in oxygenation.

\section{Conclusions}

Clinical trials in PARDS are few, and existing data highlight the need for urgent, pragmatic trials incorporating the new PALICC definition of PARDS. The PALICC definition of PARDS by providing clarity in diagnosis may aid in uniform earlier recognition of the entity, enable use of consistent management strategies and potentially increase the ease of enrollment in future clinical trials. Several valuable insights were gained from the RESTORE study; similarly, the PROSpect trial and the PAN study are expected to yield important information that could be used to design future clinical trials in PARDS. In addition, it is expected that these descriptions will be revised regularly to reflect our evolving understanding of the patho-physiology and treatment of PARDS. Furthermore, preliminary data on PARDS subtypes suggest that patient heterogeneity is an important factor in designing clinical trials and optimizing outcomes.

\section{Acknowledgments}

None.

\section{Footnote}

Conflicts of Interest: The authors have no conflicts of interest to declare.

Ethical Statement: The authors are accountable for all aspects of the work in ensuring that questions related to the accuracy or integrity of any part of the work are appropriately investigated and resolved.

\section{References}

1. Pediatric Acute Lung Injury Consensus Conference Group. Pediatric acute respiratory distress syndrome: consensus recommendations from the Pediatric Acute Lung Injury Consensus Conference. Pediatr Crit Care Med 2015;16:428-39.

2. De Luca D, van Kaam AH, Tingay DG, et al. The Montreux definition of neonatal ARDS: biological and clinical background behind the description of a new entity. 
Lancet Respir Med 2017;5:657-66.

3. Cheifetz IM. Pediatric ARDS. Respiratory care 2017;62:718-31.

4. Hough RF. Recent advances in pediatric acute respiratory distress syndrome (PARDS). Current Pediatrics Reports 2017;5:228-36.

5. Schouten LR, Veltkamp F, Bos AP, et al. Incidence and mortality of acute respiratory distress syndrome in children: a systematic review and meta-analysis. Crit Care Med 2016;44:819-29.

6. Khemani RG, Smith LS, Zimmerman JJ, et al. Pediatric acute respiratory distress syndrome: definition, incidence, and epidemiology: proceedings from the Pediatric Acute Lung Injury Consensus Conference. Pediatr Crit Care Med 2015;16:S23-40.

7. Parvathaneni K, Belani S, Leung D, et al. Evaluating the performance of the pediatric acute lung injury consensus conference definition of acute respiratory distress syndrome. Pediatr Crit Care Med 2017;18:17-25.

8. Wong JJ, Phan HP, Phumeetham S, et al. Risk stratification in pediatric acute respiratory distress syndrome: a multicenter observational study. Crit Care Med 2017;45:1820-8.

9. Wong JJ, Jit M, Sultana R, et al. Mortality in pediatric acute respiratory distress syndrome: a systematic review and meta-analysis. J Intensive Care Med 2019;34:563-71.

10. Valentine SL, Bembea MM, Muszynski JA, et al. Consensus recommendations for RBC transfusion practice in critically ill children from the pediatric critical care transfusion and anemia expertise initiative. Pediatr Crit Care Med 2018;19:884-98.

11. Brochard L, Roudot-Thoraval F, Roupie E, et al. Tidal volume reduction for prevention of ventilator-induced lung injury in acute respiratory distress syndrome. The multicenter trail group on tidal volume reduction in ARDS. Am J Respir Crit Care Med 1998;158:1831-8.

12. Stewart TE, Meade MO, Cook DJ, et al. Evaluation of a ventilation strategy to prevent barotrauma in patients at high risk for acute respiratory distress syndrome. Pressureand volume-limited ventilation strategy group. $\mathrm{N}$ Engl J Med 1998;338:355-61.

13. Acute Respiratory Distress Syndrome Network., Brower RG, Matthay MA, et al. Ventilation with lower tidal volumes as compared with traditional tidal volumes for acute lung injury and the acute respiratory distress syndrome. N Engl J Med 2000;342:1301-8.

14. Santa Cruz R, Rojas JI, Nervi R, et al. High versus low positive end-expiratory pressure (PEEP) levels for mechanically ventilated adult patients with acute lung injury and acute respiratory distress syndrome. Cochrane Database Syst Rev 2013:CD009098.

15. Briel $M$, Meade $M$, Mercat A, et al. Higher vs lower positive end-expiratory pressure in patients with acute lung injury and acute respiratory distress syndrome: systematic review and meta-analysis. JAMA 2010;303:865-73.

16. Slutsky AS, Tremblay LN. Multiple system organ failure. Is mechanical ventilation a contributing factor? Am J Respir Crit Care Med 1998;157:1721-5.

17. Tsuno K, Miura K, Takeya M, et al. Histopathologic pulmonary changes from mechanical ventilation at high peak airway pressures. Am Rev Respir Dis 1991;143:1115-20.

18. Chiumello D, Carlesso E, Brioni M, et al. Airway driving pressure and lung stress in ARDS patients. Crit Care 2016;20:276.

19. Amato MB, Meade MO, Slutsky AS, et al. Driving pressure and survival in the acute respiratory distress syndrome. $\mathrm{N}$ Engl J Med 2015;372:747-55.

20. Walkey AJ, Del Sorbo L, Hodgson CL, et al. Higher PEEP versus lower PEEP strategies for patients with acute respiratory distress syndrome. A systematic review and meta-analysis. Ann Am Thorac Soc 2017;14:S297-303.

21. Writing Group for the Alveolar Recruitment for Acute Respiratory Distress Syndrome Trial (ART) Investigators., Cavalcanti AB, Suzumura ÉA, et al. Effect of lung recruitment and titrated positive end-expiratory pressure (PEEP) vs low PEEP on mortality in patients with acute respiratory distress syndrome: a randomized clinical trial. JAMA 2017;318:1335-45.

22. Hubmayr RD. Perspective on lung injury and recruitment: a skeptical look at the opening and collapse story. Am J Respir Crit Care Med 2002;165:1647-53.

23. Rouby JJ, Brochard L. Tidal recruitment and overinflation in acute respiratory distress syndrome: yin and yang. Am J Respir Crit Care Med 2007;175:104-6.

24. Caironi P, Cressoni M, Chiumello D, et al. Lung opening and closing during ventilation of acute respiratory distress syndrome. Am J Respir Crit Care Med 2010;181:578-86.

25. Albuali WH, Singh RN, Fraser DD, et al. Have changes in ventilation practice improved outcome in children with acute lung injury? Pediatr Crit Care Med 2007;8:324-30.

26. Young D, Lamb SE, Shah S, et al. High-frequency oscillation for acute respiratory distress syndrome. $\mathrm{N} \mathrm{Engl}$ J Med 2013;368:806-13.

27. Ferguson ND, Cook DJ, Guyatt GH, et al. Highfrequency oscillation in early acute respiratory distress 
syndrome. N Engl J Med 2013;368:795-805.

28. Mora-Arteaga JA, Bernal-Ramírez OJ, Rodríguez SJ. The effects of prone position ventilation in patients with acute respiratory distress syndrome. A systematic review and metaanalysis. Med Intensiva 2015;39:359-72.

29. Lee JM, Bae W, Lee YJ, et al. The efficacy and safety of prone positional ventilation in acute respiratory distress syndrome: updated study-level meta-analysis of 11 randomized controlled trials. Crit Care Med 2014;42:1252-62.

30. Beitler JR, Shaefi S, Montesi SB, et al. Prone positioning reduces mortality from acute respiratory distress syndrome in the low tidal volume era: a meta-analysis. Intensive Care Med 2014;40:332-41.

31. Alsaghir AH, Martin CM. Effect of prone positioning in patients with acute respiratory distress syndrome: a metaanalysis. Crit Care Med 2008;36:603-9.

32. Taccone P, Pesenti A, Latini R, et al. Prone positioning in patients with moderate and severe acute respiratory distress syndrome: a randomized controlled trial. JAMA 2009;302:1977-84.

33. Guerin C, Gaillard S, Lemasson S, et al. Effects of systematic prone positioning in hypoxemic acute respiratory failure: a randomized controlled trial. JAMA 2004;292:2379-87.

34. Gattinoni L, Tognoni G, Pesenti A, et al. Effect of prone positioning on the survival of patients with acute respiratory failure. N Engl J Med 2001;345:568-73.

35. Mancebo J, Fernández R, Blanch L, et al. A multicenter trial of prolonged prone ventilation in severe acute respiratory distress syndrome. Am J Respir Crit Care Med 2006;173:1233-9.

36. Guérin C, Reignier J, Richard JC, et al. Prone positioning in severe acute respiratory distress syndrome. $\mathrm{N}$ Engl J Med 2013;368:2159-68.

37. PROSpect: prone and oscillation pediatric clinical trial. Available online: https://clinicaltrials.gov/ct2/show/ NCT03896763

38. Adhikari NK, Dellinger RP, Lundin S, et al. Inhaled nitric oxide does not reduce mortality in patients with acute respiratory distress syndrome regardless of severity: systematic review and meta-analysis. Crit Care Med 2014;42:404-12.

39. Gebistorf F, Karam O, Wetterslev J, et al. Inhaled nitric oxide for acute respiratory distress syndrome (ARDS) in children and adults. Cochrane Database Syst Rev 2016:CD002787.

40. Karam O, Gebistorf F, Wetterslev J, et al. The effect of inhaled nitric oxide in acute respiratory distress syndrome in children and adults: a Cochrane systematic review with trial sequential analysis. Anaesthesia 2017;72:106-17.

41. Taylor RW, Zimmerman JL, Dellinger RP, et al. Low-dose inhaled nitric oxide in patients with acute lung injury: a randomized controlled trial. JAMA 2004;291:1603-9.

42. Dellinger RP, Zimmerman JL, Taylor RW, et al. Effects of inhaled nitric oxide in patients with acute respiratory distress syndrome: results of a randomized phase II trial. Inhaled nitric oxide in ARDS study group. Crit Care Med 1998;26:15-23.

43. Adhikari NK, Burns KE, Friedrich JO, et al. Effect of nitric oxide on oxygenation and mortality in acute lung injury: systematic review and meta-analysis. BMJ 2007;334:779.

44. Afshari A, Brok J, Møller AM, et al. Inhaled nitric oxide for acute respiratory distress syndrome and acute lung injury in adults and children: a systematic review with meta-analysis and trial sequential analysis. Anesth Analg 2011;112:1411-21.

45. Pipeling MR, Fan E. Therapies for refractory hypoxemia in acute respiratory distress syndrome. JAMA 2010;304:2521-7.

46. Tongyoo S, Permpikul C, Mongkolpun W, et al. Hydrocortisone treatment in early sepsis-associated acute respiratory distress syndrome: results of a randomized controlled trial. Crit Care 2016;20:329.

47. Steinberg KP, Hudson LD, Goodman RB, et al. Efficacy and safety of corticosteroids for persistent acute respiratory distress syndrome. N Engl J Med 2006;354:1671-84.

48. Papazian L, Forel JM, Gacouin A, et al. Neuromuscular blockers in early acute respiratory distress syndrome. N Engl J Med 2010;363:1107-16.

49. National Heart, Lung, and Blood Institute PETAL Clinical Trials Network., Moss M, Huang DT, et al. Early neuromuscular blockade in the acute respiratory distress syndrome. N Engl J Med 2019;380:1997-2008.

50. Paediatric ards neuromuscular blockade study (PAN). Available online: https:/clinicaltrials.gov/ct2/show/ NCT02902055

51. Curley MA, Wypij D, Watson RS, et al. Protocolized sedation vs usual care in pediatric patients mechanically ventilated for acute respiratory failure: a randomized clinical trial. JAMA 2015;313:379-89.

52. National Heart, Lung, and Blood Institute Acute Respiratory Distress Syndrome (ARDS) Clinical Trials Network., Wiedemann HP, Wheeler AP, et al. Comparison of two fluid-management strategies in acute lung injury. $\mathrm{N}$ 
Engl J Med 2006;354:2564-75.

53. Chu DK, Kim LH, Young PJ, et al. Mortality and morbidity in acutely ill adults treated with liberal versus conservative oxygen therapy (IOTA): a systematic review and meta-analysis. Lancet 2018;391:1693-705.

54. Peters MJ, Jones GAL, Wiley D, et al. Conservative versus liberal oxygenation targets in critically ill children: the randomised multiple-centre pilot Oxy-PICU trial. Intensive Care Med 2018;44:1240-8.

55. Talmor D, Sarge T, Malhotra A, et al. Mechanical ventilation guided by esophageal pressure in acute lung injury. N Engl J Med 2008;359:2095-104.

56. Fish E, Novack V, Banner-Goodspeed VM, et al. The esophageal pressure-guided ventilation 2 (EPVent2) trial protocol: a multicentre, randomised clinical trial of mechanical ventilation guided by transpulmonary pressure. BMJ Open 2014;4:e006356.

57. Calfee CS, Delucchi K, Parsons PE, et al. Subphenotypes in acute respiratory distress syndrome: latent class analysis of data from two randomised controlled trials. Lancet Respir Med 2014;2:611-20.

58. Calfee CS, Janz DR, Bernard GR, et al. Distinct molecular phenotypes of direct vs indirect ARDS in single-center and multicenter studies. Chest 2015;147:1539-48.

59. Jabaudon M, Blondonnet R, Audard J, et al. Recent directions in personalised acute respiratory distress syndrome medicine. Anaesth Crit Care Pain Med 2018;37:251-8.

60. Flori H, Sapru A, Quasney MW, et al. A prospective investigation of interleukin-8 levels in pediatric acute respiratory failure and acute respiratory distress syndrome. Crit Care 2019;23:128.

61. Yehya N, Keim G, Thomas NJ. Subtypes of pediatric acute respiratory distress syndrome have different predictors of mortality. Intensive Care Med 2018;44:1230-9.

62. Identifying PARDS endotypes. Available online: https:// clinicaltrials.gov/ct2/show/NCT03539783
Cite this article as: Allareddy V, Cheifetz IM. Clinical trials and future directions in pediatric acute respiratory distress syndrome. Ann Transl Med 2019;7(19):514. doi: 10.21037/ atm.2019.09.14 